\title{
RUIN IN THE PERTURBED \\ COMPOUND POISSON RISK PROCESS UNDER INTEREST FORCE
}

\author{
JUN CAI, ${ }^{*}$ University of Waterloo \\ HAILIANG YANG, ${ }^{* *}$ The University of Hong Kong
}

\begin{abstract}
In this paper, we study ruin in a perturbed compound Poisson risk process under stochastic interest force and constant interest force. By using the technique of stochastic control, we show that the ruin probability in the perturbed risk model is always twice continuously differentiable provided that claim sizes have continuous density functions. In the perturbed risk model, ruin may be caused by a claim or by oscillation. We decompose the ruin probability into the sum of two ruin probabilities; one is the probability that ruin is caused by a claim and the other is the probability that ruin is caused by oscillation. Integrodifferential equations for these ruin probabilities are derived when the interest force is constant. When the claim sizes are exponentially distributed, explicit solutions of the ruin probabilities are derived from the integrodifferential equations. Numerical examples are given to illustrate the effects of diffusion volatility and interest force on the ruin probabilities.
\end{abstract}

Keywords: Compound Poisson risk process; diffusion; Brownian motion; jump diffusion process; ruin probability; Hamilton-Jacobi-Bellman equation; viscosity solution; confluent hypergeometric function; Kummer's confluent hypergeometric equation

2000 Mathematics Subject Classification: Primary 60J75

Secondary 60J65; 91B30

\section{Introduction}

Let

$$
U_{t}=c t-\sum_{k=1}^{N(t)} Y_{k}+\sigma W_{t}=c t-S_{t}+\sigma W_{t}, \quad t \geq 0,
$$

be a compound Poisson risk process perturbed by a diffusion or a jump diffusion process, where $c>0$ is the rate of premium; $\left\{Y_{k}, k=1,2, \ldots\right\}$ is a sequence of independent and identically distributed nonnegative random variables, denoting claim sizes; $\{N(t), t \geq 0\}$ is a Poisson process with rate $\lambda>0$, representing the number of claims up to time $t ;\left\{W_{t}, t \geq 0\right\}$ is a standard Brownian motion; $\sigma>0$ is a constant, representing the diffusion volatility parameter; and $S_{t}=\sum_{k=1}^{N(t)} Y_{k}$ is the compound Poisson process. In addition, $\left\{Y_{k}, k=1,2, \ldots\right\}$, $\{N(t), t \geq 0\}$, and $\left\{W_{t}, t \geq 0\right\}$ are independent. As pointed out in Dufresne and Gerber (1991), the perturbed compound Poisson risk process adds an uncertainty to premium income or an additional uncertainty to aggregate claims.

Received 9 February 2005; revision received 8 May 2005.

* Postal address: Department of Statistics and Actuarial Science, University of Waterloo, Waterloo, Ontario, Canada N2L 3G1. Email address: jcai@math.uwaterloo.ca

** Postal address: Department of Statistics and Actuarial Science, The University of Hong Kong, Pokfulam Road, Hong Kong. Email address: hlyang@hkusua.hku.hk 
Assume that the surplus $U_{t}$ is invested continuously at a constant interest force $\delta>0$ and let $X_{t}$ denote the surplus at time $t$ under the constant interest force $\delta$ with an initial surplus $u \geq 0$; thus,

$$
X_{t}=\mathrm{e}^{\delta t}\left(u+\int_{0}^{t} \mathrm{e}^{-\delta s} \mathrm{~d} U_{s}\right), \quad t \geq 0, \quad X_{0}=u .
$$

More generally, if the surplus $U_{t}$ is invested continuously at a stochastic interest force and the deterministic function $\delta t$ in (1.2) is another jump diffusion process $\delta_{t}$ with

$$
\delta_{t}=\delta t+\sigma_{\mathrm{R}} W_{\mathrm{R}, t}+\sum_{i=1}^{N_{\mathrm{R}}(t)} \ln \left(1+X_{i}\right)=\delta t+\sigma_{\mathrm{R}} W_{\mathrm{R}, t}+S_{\mathrm{R}, t}
$$

or, equivalently, the deterministic function $\mathrm{e}^{\delta t}$ in (1.2) is replaced by a stochastic process $\mathrm{e}^{\delta_{t}}$ given by

$$
\mathrm{e}^{\delta_{t}}=\mathrm{e}^{\delta t+\sigma_{\mathrm{R}} W_{\mathrm{R}, t}} \prod_{i=1}^{N_{\mathrm{R}}(t)}\left(1+X_{i}\right),
$$

then the surplus at time $t$ under the stochastic interest force $\delta_{t}$ with an initial surplus $u \geq 0$ is given by

$$
X_{t}=\mathrm{e}^{\delta_{t}}\left(u+\int_{0}^{t} \mathrm{e}^{-\delta_{s}} \mathrm{~d} U_{s}\right), \quad t \geq 0, \quad X_{0}=u .
$$

Here $\sigma_{\mathrm{R}}$ is a constant; $\left\{W_{\mathrm{R}, t}, t \geq 0\right\}$ is a standard Brownian motion; $\left\{N_{\mathrm{R}}(t), t \geq 0\right\}$ is a Poisson process with rate $\lambda_{\mathrm{R}} \geq 0,\left\{X_{i}, i=1,2, \ldots\right\}$ is a sequence of independent and identically distributed random variables such that $1+X_{i}>0$ for all $i=1,2, \ldots ;\left\{W_{\mathrm{R}, t}, t \geq 0\right\}$, $\left\{N_{\mathrm{R}}(t), t \geq 0\right\}$, and $\left\{X_{i}, i=1,2, \ldots\right\}$ are independent; and $S_{\mathrm{R}, t}=\sum_{i=1}^{N_{\mathrm{R}}(t)} \ln \left(1+X_{i}\right)$ is a compound Poisson process. Furthermore, the process $\left\{\delta_{t}, t \geq 0\right\}$ is assumed to be independent of the process $\left\{U_{t}, t \geq 0\right\}$ and, by convention, $\sum_{i=1}^{0}=0$ and $\prod_{i=1}^{0}=1$.

The risk process (1.3) has been studied by Paulsen and Gjessing (1997) and expressed in the form of their Equation (2.4). The risk process (1.2) is a special case of the risk process (1.3) when $\sigma_{\mathrm{R}}=0$ and $\lambda_{\mathrm{R}}=0$.

Denote the ruin time of the risk process (1.3) by $T$, i.e. $T=\inf \left\{t: X_{t}<0\right\}$ or $T=\infty$ if $X_{t} \geq 0$ for all $t \geq 0$. Define the ruin probability with an initial surplus $u \geq 0$ by

$$
\psi(u)=\operatorname{Pr}\left\{T<\infty \mid X_{0}=u\right\}=\operatorname{Pr}\left\{X_{t}<0 \text { for some } t \geq 0 \mid X_{0}=u\right\} .
$$

In this perturbed risk model, ruin may occur in two different situations. In one situation, ruin is caused by a claim and, in the other, ruin is caused by oscillation. We let

$$
T_{\mathrm{S}}=\left\{\begin{array}{l}
\inf \left\{t: X_{t}<0, X_{h}>0,0<h<t\right\} \\
\infty \quad \text { if } X_{t} \geq 0 \text { for all } t \geq 0
\end{array}\right.
$$

i.e. $T_{\mathrm{S}}$ is the time at which ruin is caused by a claim. Furthermore, we let

$$
T_{\mathrm{d}}=\left\{\begin{array}{l}
\inf \left\{t: X_{t}=0, X_{h}>0,0<h<t\right\} \\
\infty \quad \text { if } X_{t} \geq 0 \text { for all } t \geq 0
\end{array}\right.
$$


i.e. $T_{\mathrm{d}}$ is the time at which ruin is caused by oscillation. Then $T=\min \left\{T_{\mathrm{s}}, T_{\mathrm{d}}\right\}$. Moreover, we denote the ruin probabilities in the two situations by

$$
\psi_{\mathrm{s}}(u)=\operatorname{Pr}\left\{T_{\mathrm{s}}<\infty \mid X_{0}=u\right\}
$$

and

$$
\psi_{\mathrm{d}}(u)=\operatorname{Pr}\left\{T_{\mathrm{d}}<\infty \mid X_{0}=u\right\}
$$

respectively.

It is obvious that the ruin probability $\psi(u)$ can be decomposed as follows:

$$
\psi(u)=\psi_{\mathrm{s}}(u)+\psi_{\mathrm{d}}(u), \quad u \geq 0 .
$$

In addition, it follows from the oscillating nature of the sample paths of $X_{t}$ that

$$
\psi_{\mathrm{d}}(0)=\psi(0)=1 \quad \text { and } \quad \psi_{\mathrm{s}}(0)=0
$$

See Dufresne and Gerber (1991) for a detailed discussion of (1.4) and (1.5) in the perturbed compound Poisson risk process (1.1).

Given that ruin has occurred, the probability that it is due to a claim is given by $\operatorname{Pr}\left\{T_{\mathrm{S}}<\infty \mid T<\infty\right\}=\psi_{\mathrm{s}}(u) / \psi(u)$ and the probability that it is due to oscillation is given by $\operatorname{Pr}\left\{T_{\mathrm{d}}<\infty \mid T<\infty\right\}=\psi_{\mathrm{d}}(u) / \psi(u)$.

Ruin in the perturbed compound Poisson process has been studied extensively in the literature; see, for example, Dufresne and Gerber (1991), Gerber and Landry (1998), Paulsen and Gjessing (1997), Yang and Zhang (2001), and references therein. Recently, ruin under interest force has attracted increasing attention in the risk theory literature. For instance, ruin in the compound Poisson risk process under constant interest force has been studied in Asmussen (2000), Cai and Dickson (2002), Paulsen and Gjessing (1997), Sundt and Teugels (1995), and references therein.

In the study of ruin probability, a commonly used method is to first derive integrodifferential or differential equations satisfied by the ruin probability and then to discuss the properties and solutions of the ruin probability based on the equations. For example, let $\phi(u)$ be the ruin probability in the perturbed compound Poisson process without interest force or in the risk process (1.1), i.e. $\phi(u)=\operatorname{Pr}\left\{u+U_{t}<0\right.$ for some $\left.t \geq 0\right\}$. Using a heuristic argument, Dufresne and Gerber (1991) derived an integrodifferential equation (their Equation (2.1)) for $\phi(u)$ under the assumption that $\phi(u)$ is twice continuously differentiable. The same argument and assumption were used in Gerber and Landry (1998), and references therein, in another study of ruin in the risk process (1.1). However, these references did not explain under what conditions $\phi(u)$ is twice continuously differentiable.

For the ruin probability $\psi(u)$ in the general risk process (1.3), Paulsen and Gjessing (1997) derived, in their Theorem 2.1, the integrodifferential equation for $\psi(u)$ by using Itô's formula under the assumption that $\psi(u)$ is twice continuously differentiable with a bounded first derivative. Again, Paulsen and Gjessing (1997) did not discuss under what conditions $\psi(u)$ is twice continuously differentiable.

Theoretically, it is necessary to discuss the differentiability of the ruin probability when one derives integrodifferential or differential equations for it. Such a discussion for the ruin probability in the compound Poisson risk process can be found in Grandell (1991), who used renewal and differential arguments. Furthermore, Wang and Wu (2001) and Cai (2004) have studied the differentiability of the ruin probability $\psi(u)$ in the risk process $(1.3)$ when $\lambda_{\mathrm{R}}=0$. 
They first used a renewal argument to derive the integral equations for $\psi(u)$, and then gave the conditions under which $\psi(u)$ is twice, or more times, continuously differentiable. Their conditions involve the differentiability of the density function of the claim size $Y_{1}$ and relationships between the parameters $\lambda, \delta$, and $\sigma$. Unfortunately, neither the methods of Wang and $\mathrm{Wu}$ (2001) and Cai (2004) nor those of Grandell (1991) apply to the differentiability of the ruin probability in (1.3) in general cases.

In this paper, we first use the technique of stochastic control and the result of Hipp and Plum (2003) to prove that the ruin probability $\psi(u)$ in the risk process (1.3) is always twice continuously differentiable, provided that the claim size $Y_{1}$ has a continuous density function, and to give the integrodifferential equation for $\psi(u)$. We then discuss ruin probabilities in the perturbed compound Poisson risk process with constant interest force in detail. In this case, we derive integrodifferential equations for the ruin probability $\psi(u)$ using the HamiltonJacobi-Bellman (HJB) equation and the ruin probabilities $\psi_{\mathrm{s}}(u)$ and $\psi_{\mathrm{d}}(u)$ using a differential argument and the Itô formula. When claim sizes are exponentially distributed, explicit solutions of these ruin probabilities are given. Numerical examples are given to illustrate these results and the effects of diffusion volatility and interest force on the ruin probabilities.

\section{The HJB equation and differentiability of the ruin probability}

In this section, using stochastic control techniques and the result of Hipp and Plum (2003), we prove that the ruin probability $\psi(u)$ in the risk process (1.3) is always twice continuously differentiable provided that the claim sizes have a continuous density function.

We first describe the ideas of the proof. It is well known in stochastic control theory that, in many cases, a value function is not smooth enough to satisfy the HJB equation in the classical or usual sense. A weak formulation of the solution to the HJB equation has been used in the literature. A commonly used weak formulation is called the viscosity solution, proposed by Crandall and Lions (1983). For more detailed discussions of viscosity solutions, see Crandall et al. (1992) and Fleming and Soner (1993). Here, by following the paper of Hipp and Plum (2003), we first show that the survival probability in the risk process (1.3) satisfies the HJB equation in a weak formulation sense. It is well known that, as in Hipp and Plum (2003), the HJB equation has a unique solution. Thus, the solution must be the survival probability in our problem. We then use the result in Hipp and Plum (2003), which states that the solution to the corresponding HJB equation is twice continuously differentiable. Therefore, the ruin probability, as a function of the initial surplus, is twice continuously differentiable.

We then give the detailed arguments of the proof. In doing so, we describe the risk model (1.3) in terms of stochastic differential equations, as follows. The dynamics of the surplus process $X_{t}$ in (1.3) can be expressed as

$$
\begin{aligned}
\mathrm{d} X_{t} & =\left(c+\delta_{t} X_{t}\right) \mathrm{d} t+\sigma \mathrm{d} W_{t}-\mathrm{d} S_{t}, & X_{0} & =u, \\
\mathrm{~d} \delta_{t} & =\delta \mathrm{d} t+\sigma_{\mathrm{R}} \mathrm{d} W_{\mathrm{R}, t}+\mathrm{d} S_{\mathrm{R}, t}, & \delta_{0} & =\delta_{0} .
\end{aligned}
$$

Instead of considering the original problem, we first consider the following problem. We assume that an insurance company pays dividends continuously at a rate of $\alpha\left(X_{\alpha}(t)\right)$ at time $t$. Suppose that the dynamics of the surplus process $X_{\alpha}(t)$ is given by

$$
\begin{aligned}
\mathrm{d} X_{\alpha}(t) & =\left(c+\delta_{t} X_{\alpha}(t)-\alpha\left(X_{\alpha}(t)\right)\right) \mathrm{d} t+\sigma \mathrm{d} W_{t}-\mathrm{d} S_{t}, & X_{\alpha}(0) & =u, \\
\mathrm{~d} \delta_{t} & =\delta \mathrm{d} t+\sigma_{\mathrm{R}} \mathrm{d} W_{\mathrm{R}, t}+\mathrm{d} S_{\mathrm{R}, t}, & \delta_{0} & =\delta_{0} .
\end{aligned}
$$


Assume that $Y_{1}$ and $X_{1}$ have finite expectations and continuous distributions. We know that the surplus process $X_{\alpha}(t)$ has the same distribution as $\hat{X}_{\alpha}(t)$, where the surplus process $\hat{X}_{\alpha}(t)$ is given by

$$
\begin{aligned}
\mathrm{d} \hat{X}_{\alpha}(t) & =\left(c+\delta_{t} \hat{X}_{\alpha}(t)-\alpha\left(\hat{X}_{\alpha}(t)\right)\right) \mathrm{d} t+\sqrt{\sigma^{2}+\sigma_{\mathrm{R}}^{2} \hat{X}_{\alpha}^{2}(t)} \mathrm{d} B_{t}-\mathrm{d} S_{t}+\hat{X}_{\alpha}\left(t^{-}\right) \mathrm{d} S_{\mathrm{R}, t}, \\
\hat{X}_{\alpha}(0) & =u
\end{aligned}
$$

cf. Equation (2.5) of Paulsen and Gjessing (1997). Here $\left\{B_{t}, t \geq 0\right\}$ is a standard Brownian motion independent of the compound Poisson processes $\left\{S_{t}, t \geq 0\right\}$ and $\left\{S_{\mathrm{R}, t}, t \geq 0\right\}$.

As before, we define the ruin probability associated with the risk process (2.2) as

$$
\psi_{\alpha}(u)=\operatorname{Pr}\left\{\hat{X}_{\alpha}(t)<0 \text { for some } t \geq 0 \mid \hat{X}_{\alpha}(0)=u\right\} .
$$

The objective of the insurance company is to minimize the ruin probability $\psi_{\alpha}(u)$ by choosing the optimal dividend strategy. This is a stochastic control problem. Let $R_{\alpha}(u)=1-\psi_{\alpha}(u)$ be the survival probability corresponding to dividend policy $\alpha$. Then, by the dynamic programming principle, the value function $R_{\alpha}(u)$ satisfies the following HJB equation, where a prime denotes differentiation:

$$
\begin{gathered}
\sup _{\alpha}\left\{\lambda \mathrm{E}\left[R_{\alpha}\left(u-Y_{1}\right)-R_{\alpha}(u)\right]+\lambda_{\mathrm{R}} \mathrm{E}\left[R_{\alpha}\left(u\left(1+X_{1}\right)\right)-R_{\alpha}(u)\right]\right. \\
\left.+(c+\delta u-\alpha(u)) R_{\alpha}^{\prime}(u)+\frac{1}{2}\left(\sigma^{2}+u^{2} \sigma_{\mathrm{R}}^{2}\right) R_{\alpha}^{\prime \prime}(u)\right\}=0 .
\end{gathered}
$$

It is obvious that the optimal dividend policy is for the insurance company to pay no dividends, or to set $\alpha=0$. By the verification theorem, the HJB equation (2.3) becomes

$$
\begin{gathered}
\lambda \mathrm{E}\left[R_{0}\left(u-Y_{1}\right)-R_{0}(u)\right]+\lambda_{\mathrm{R}} \mathrm{E}\left[R_{0}\left(u\left(1+X_{1}\right)\right)-R_{0}(u)\right] \\
+(c+\delta u) R_{0}^{\prime}(u)+\frac{1}{2}\left(\sigma^{2}+u^{2} \sigma_{\mathrm{R}}^{2}\right) R_{0}^{\prime \prime}(u)=0 .
\end{gathered}
$$

As mentioned earlier, it is well known that (2.4) has a unique continuous solution. Furthermore, $R_{0}(u)$ is the survival probability of the surplus process $\hat{X}_{0}(t)=\hat{X}(t)$ given by

$$
\begin{aligned}
\mathrm{d} \hat{X}(t) & =\left(c+\delta_{t} \hat{X}(t)\right) \mathrm{d} t+\sqrt{\sigma^{2}+\sigma_{\mathrm{R}}^{2} \hat{X}^{2}(t)} \mathrm{d} B_{t}-\mathrm{d} S_{t}+\hat{X}\left(t^{-}\right) \mathrm{d} S_{\mathrm{R}, t}, \\
\hat{X}(0) & =u
\end{aligned}
$$

Again, $\hat{X}(t)$ and $X_{t}$ in (2.1) have the same distribution and, therefore, $R_{0}(u)$ and $\psi_{0}(u)$ are respectively the survival and ruin probabilities for model (2.1). Hence, $R_{0}(u)=R(u)$ and $\psi_{0}(u)=\psi(u)$.

We have the following result, similar to Theorem 5 of Hipp and Plum (2003), showing that $R(u)=1-\psi(u)$ is twice continuously differentiable.

Theorem 2.1. Assume that the claim size $Y_{1}$ has a continuous density $f(y)$. Then (2.4) has a unique solution $R(u)$ that is nonnegative, nondecreasing, concave with $R(\infty)=1$, continuous on $[0, \infty)$, and twice continuously differentiable on $(0, \infty)$.

Proof. Equation (2.4) has a form similar to Equation (10) of Hipp and Plum (2003), and the proof of Theorem 5 of Hipp and Plum (2003) applies to it. We thus omit the proof of Theorem 2.1, and refer the reader to Hipp and Plum (2003) for details. 
Remark 2.1. Paulsen and Gjessing (1997) assumed that $\psi(u)$ is twice continuously differentiable in order to use Itô's formula to obtain the integrodifferential equation satisfied by $\psi(u)$. Here, using the result of Hipp and Plum (2003), we prove that $\psi(u)$ is always twice continuously differentiable provided that the claim sizes have continuous density functions. This result also greatly relaxes the conditions of Wang and Wu (2001) and Cai (2004) on differentiability of the ruin probability $\psi(u)$.

\section{Integrodifferential equations for ruin probabilities}

In this section, we study the risk process $X_{t}$ given in (1.2) and consider ruin in the perturbed compound Poisson risk process under constant interest force. We first give the integrodifferential equation for $\psi(u)$ using the HJB equation (2.4) directly and then, using a differential argument and Itô's formula, we derive integrodifferential equations for $\psi_{\mathrm{s}}(u)$ and $\psi_{\mathrm{d}}(u)$. The differential argument is a common method used in ruin theory; see Grandell (1991) for the method used in the compound Poisson risk process and Dufresne and Gerber (1991) for the method used in the perturbed compound Poisson risk process.

Throughout the paper, we denote the distribution function of $Y_{1}$ by $F$, with $F(0)=0$, and the tail of a distribution function $B$ by $\bar{B}(x)=1-B(x)$.

Theorem 3.1. (Integrodifferential equation for $\psi(u)$.) Assume that $F$ has a continuous density function. Then, for any $u>0, \psi(u)$ satisfies the integrodifferential equation

$$
\frac{1}{2} \sigma^{2} \psi^{\prime \prime}(u)+(\delta u+c) \psi^{\prime}(u)+\lambda \bar{F}(u)=\lambda \psi(u)-\lambda \int_{0}^{u} \psi(u-y) \mathrm{d} F(y)
$$

with the following boundary conditions:

$$
\psi(\infty)=0, \quad \psi(0)=1, \quad \frac{1}{2} \sigma^{2} \psi^{\prime \prime}\left(0^{+}\right)+c \psi^{\prime}\left(0^{+}\right)=0 .
$$

Proof. Let $\lambda_{\mathrm{R}}=0$ and $\sigma_{\mathrm{R}}=0$ and note that $R_{0}(u)=1-\psi_{0}(u)=1-\psi(u)$ in the HJB equation (2.4). We then have

$$
\lambda \psi(u)-\lambda \mathrm{E}\left[\psi\left(u-Y_{1}\right)\right]=(c+\delta u) \psi^{\prime}(u)+\frac{1}{2} \sigma^{2} \psi^{\prime \prime}(u) .
$$

Furthermore,

$$
\begin{aligned}
\mathrm{E}\left[\psi\left(u-Y_{1}\right)\right] & =\int_{0}^{\infty} \psi(u-y) \mathrm{d} F(y) \\
& =\int_{0}^{u} \psi(u-y) \mathrm{d} F(y)+\int_{u}^{\infty} \psi(u-y) \mathrm{d} F(y) \\
& =\int_{0}^{u} \psi(u-y) \mathrm{d} F(y)+\bar{F}(u),
\end{aligned}
$$

which, together with (3.3), implies (3.1).

In addition, the boundary condition $\psi(\infty)=0$ follows from $\psi(u) \leq \phi(u)$ and $\phi(\infty)=0$, the boundary condition $\psi(0)=1$ follows from (1.5), and the last boundary condition follows from letting $u \downarrow 0$ in (3.1).

We point out that (3.1) can also be derived using Theorem 2.1 of Paulsen and Gjessing (1997). However, their theorem holds under the assumption that $\psi(u)$ is twice continuously differentiable and has a bounded first derivative. 
Furthermore, we point out that the arguments in Section 2 for the differentiability of $\psi(u)$ do not apply to $\psi_{\mathrm{s}}(u)$ and $\psi_{\mathrm{d}}(u)$ and, hence, that HJB equations similar to (2.4) are not available for $\psi_{\mathrm{s}}(u)$ and $\psi_{\mathrm{d}}(u)$. The reason is that, to use the stochastic control method, we must have a system of stochastic differential equations for $\psi(u)$ : the corresponding systems of stochastic differential equations for $\psi_{\mathrm{s}}(u)$ and $\psi_{\mathrm{d}}(u)$ are unknown. In other words, we cannot separate the system of stochastic differential equations for $\psi(u)$ into two parts.

However, using a differential argument and Itô's formula, we can derive integrodifferential equations for $\psi_{\mathrm{s}}(u)$ and $\psi_{\mathrm{d}}(u)$ under the assumption that both $\psi_{\mathrm{s}}(u)$ and $\psi_{\mathrm{d}}(u)$ are twice continuously differentiable. We conjecture that $\psi_{\mathrm{s}}(u)$ and $\psi_{\mathrm{d}}(u)$ are twice continuously differentiable provided that $F$ has a continuous density function, as we did for $\psi(u)$.

Theorem 3.2. (Integrodifferential equation for $\psi_{\mathrm{s}}(u)$.) Assume that $\psi_{\mathrm{s}}(u)$ is twice continuously differentiable. Then, for any $u>0, \psi_{\mathrm{s}}(u)$ satisfies the integrodifferential equation

$$
\frac{1}{2} \sigma^{2} \psi_{\mathrm{s}}^{\prime \prime}(u)+(\delta u+c) \psi_{\mathrm{s}}^{\prime}(u)+\lambda \bar{F}(u)=\lambda \psi_{\mathrm{s}}(u)-\lambda \int_{0}^{u} \psi_{\mathrm{s}}(u-y) \mathrm{d} F(y)
$$

with the following boundary conditions:

$$
\psi_{\mathrm{s}}(\infty)=0, \quad \psi_{\mathrm{s}}(0)=0, \quad \frac{1}{2} \sigma^{2} \psi_{\mathrm{s}}^{\prime \prime}\left(0^{+}\right)+c \psi_{\mathrm{s}}^{\prime}\left(0^{+}\right)=-\lambda
$$

Proof. Let

$$
h(t)=u \mathrm{e}^{\delta t}+c \int_{0}^{t} \mathrm{e}^{\delta s} \mathrm{~d} s+\sigma \int_{0}^{t} \mathrm{e}^{\delta s} \mathrm{~d} W_{s}-u .
$$

Consider the risk process $X_{t}$, defined by (1.2), in an infinitesimal time interval $(0, t]$. Since $N(t)$ is a Poisson process, there are three possible cases.

(i) There are no claims in $(0, t]$ and, thus,

$$
X_{t}=u \mathrm{e}^{\delta t}+c \int_{0}^{t} \mathrm{e}^{\delta s} \mathrm{~d} s+\sigma \int_{0}^{t} \mathrm{e}^{\delta s} \mathrm{~d} W_{s}=u+h(t)
$$

(ii) There is exactly one claim in $(0, t]$, with claim amount $y$, and

(a) $y<u+h(t)$, i.e. ruin does not occur and, thus,

$$
X_{t}=u \mathrm{e}^{\delta t}+c \int_{0}^{t} \mathrm{e}^{\delta s} \mathrm{~d} s+\sigma \int_{0}^{t} \mathrm{e}^{\delta s} \mathrm{~d} W_{s}-y=u+h(t)-y
$$

(b) $y>u+h(t)$, i.e. ruin occurs due to the claim; or

(c) $y=u+h(t)$, i.e. ruin occurs due to oscillation (the probability that this case occurs is 0 ).

(iii) There is more than one claim in $(0, t]$. 
Thus, considering cases (i), (ii), and (iii) and noticing that, in case (ii)(b), $\psi_{\mathrm{s}}(u+h(t)-y)=1$ if $y>u+h(t)$, we have

$$
\begin{aligned}
\psi_{\mathrm{S}}(u)= & (1-\lambda t) \mathrm{E}\left[\psi_{\mathrm{s}}(u+h(t))\right]+\lambda t \mathrm{E}\left[\int_{0}^{u+h(t)} \psi_{\mathrm{s}}(u+h(t)-y) \mathrm{d} F(y)\right] \\
& +\lambda t \mathrm{E}\left[\int_{u+h(t)}^{\infty} \psi_{\mathrm{s}}(u+h(t)-y) \mathrm{d} F(y)\right]+o(t) \\
= & (1-\lambda t) \mathrm{E}\left[\psi_{\mathrm{s}}(u+h(t))\right]+\lambda t \mathrm{E}\left[\int_{0}^{u+h(t)} \psi_{\mathrm{s}}(u+h(t)-y) \mathrm{d} F(y)\right] \\
& +\lambda t \mathrm{E}[\bar{F}(u+h(t))]+o(t)
\end{aligned}
$$

or, equivalently,

$$
\begin{aligned}
\lambda t \mathrm{E}\left[\psi_{\mathrm{s}}(u+h(t))\right]= & \mathrm{E}\left[\psi_{\mathrm{s}}(u+h(t))\right]-\psi_{\mathrm{s}}(u)+\lambda t \mathrm{E}\left[\int_{0}^{u+h(t)} \psi_{\mathrm{s}}(u+h(t)-y) \mathrm{d} F(y)\right] \\
& +\lambda t \mathrm{E}[\bar{F}(u+h(t))]+o(t) .
\end{aligned}
$$

If we let $Y(t)=u+h(y)$ then (3.6) implies that

$$
\mathrm{d} Y(t)=(u \delta+c) \mathrm{e}^{\delta t} \mathrm{~d} t+\sigma \mathrm{e}^{\delta t} \mathrm{~d} W_{t}, \quad Y(0)=u .
$$

By Itô's formula, we have

$$
\begin{aligned}
\mathrm{d} \psi_{\mathrm{s}}(u+h(t)) & =\mathrm{d} \psi_{\mathrm{s}}(Y(t)) \\
& =\left((u \delta+c) \mathrm{e}^{\delta t} \psi_{\mathrm{s}}^{\prime}(Y(t))+\frac{1}{2} \sigma^{2} \mathrm{e}^{2 \delta t} \psi_{\mathrm{s}}^{\prime \prime}(Y(t))\right) \mathrm{d} t+\sigma \mathrm{e}^{\delta t} \psi_{\mathrm{s}}^{\prime}(Y(t)) \mathrm{d} W_{t}
\end{aligned}
$$

or, equivalently,

$$
\begin{aligned}
\psi_{\mathrm{s}}(u+h(t))= & \psi_{\mathrm{s}}(Y(t)) \\
= & \psi_{\mathrm{s}}(u)+\int_{0}^{t}\left((u \delta+c) \mathrm{e}^{\delta x} \psi_{\mathrm{s}}^{\prime}(Y(x))+\frac{1}{2} \sigma^{2} \mathrm{e}^{2 \delta x} \psi_{\mathrm{s}}^{\prime \prime}(Y(x))\right) \mathrm{d} x \\
& +\int_{0}^{t} \sigma \mathrm{e}^{\delta x} \psi_{\mathrm{s}}^{\prime}(Y(x)) \mathrm{d} W_{x},
\end{aligned}
$$

which implies that

$$
\begin{aligned}
& \mathrm{E}\left[\psi_{\mathrm{s}}(u+h(t))\right] \\
& \quad=\psi_{\mathrm{s}}(u)+\int_{0}^{t}\left((u \delta+c) \mathrm{e}^{\delta x} \mathrm{E}\left[\psi_{\mathrm{s}}^{\prime}(Y(x))\right]+\frac{1}{2} \sigma^{2} \mathrm{e}^{2 \delta x} \mathrm{E}\left[\psi_{\mathrm{s}}^{\prime \prime}(Y(x))\right]\right) \mathrm{d} x .
\end{aligned}
$$

Therefore, by dividing by $t$ on both sides of (3.7), letting $t \rightarrow 0$, and using (3.8), we obtain

$$
\lambda \psi_{\mathrm{s}}(u)=(\delta u+c) \psi_{\mathrm{s}}^{\prime}(u)+\frac{1}{2} \sigma^{2} \psi_{\mathrm{s}}^{\prime \prime}(u)+\lambda \int_{0}^{u} \psi_{\mathrm{s}}(u-y) \mathrm{d} F(y)+\lambda \bar{F}(u),
$$

which implies (3.4).

In addition, the boundary condition $\psi_{\mathrm{s}}(\infty)=0$ follows from $\psi_{\mathrm{s}}(u) \leq \psi(u)$ and $\psi(\infty)=0$, the boundary condition $\psi_{\mathrm{s}}(0)=0$ follows from (1.5), and the last boundary condition follows from letting $u \downarrow 0$ in (3.4). 
We note that $\psi(u)$ and $\psi_{\mathrm{s}}(u)$ satisfy the same second-order integrodifferential equations, but have different boundary conditions.

Theorem 3.3. (Integrodifferential equation for $\psi_{\mathrm{d}}(u)$.) Assume that $\psi_{\mathrm{d}}(u)$ is twice continuously differentiable. Then, for any $u>0, \psi_{\mathrm{d}}(u)$ satisfies the integrodifferential equation

$$
\frac{1}{2} \sigma^{2} \psi_{\mathrm{d}}^{\prime \prime}(u)+(\delta u+c) \psi_{\mathrm{d}}^{\prime}(u)=\lambda \psi_{\mathrm{d}}(u)-\lambda \int_{0}^{u} \psi_{\mathrm{d}}(u-y) \mathrm{d} F(y)
$$

with the following boundary conditions:

$$
\psi_{\mathrm{d}}(\infty)=0, \quad \psi_{\mathrm{d}}(0)=1, \quad \frac{1}{2} \sigma^{2} \psi_{\mathrm{d}}^{\prime \prime}\left(0^{+}\right)+c \psi_{\mathrm{d}}^{\prime}\left(0^{+}\right)=\lambda .
$$

Proof. Consider cases (i), (ii), and (iii) in the proof of Theorem 3.2 and notice that, in case (ii)(b), $\psi_{\mathrm{d}}(u+h(t)-y)=0$ if $y>u+h(t)$. We see that

$$
\begin{aligned}
\psi_{\mathrm{d}}(u)= & (1-\lambda t) \mathrm{E}\left[\psi_{\mathrm{d}}(u+h(t))\right]+\lambda t \mathrm{E}\left[\int_{0}^{u+h(t)} \psi_{\mathrm{d}}(u+h(t)-y) \mathrm{d} F(y)\right] \\
& +\lambda t \mathrm{E}\left[\int_{u+h(t)}^{\infty} \psi_{\mathrm{d}}(u+h(t)-y) \mathrm{d} F(y)\right]+o(t) \\
= & (1-\lambda t) \mathrm{E}\left[\psi_{\mathrm{d}}(u+h(t))\right]+\lambda t \mathrm{E}\left[\int_{0}^{u+h(t)} \psi_{\mathrm{d}}(u+h(t)-y) \mathrm{d} F(y)\right]+o(t),
\end{aligned}
$$

which implies (3.9) by the same arguments as for (3.1).

In addition, the boundary condition $\psi_{\mathrm{d}}(\infty)=0$ follows from $\psi_{\mathrm{d}}(u) \leq \psi(u)$ and $\psi(\infty)=0$, the boundary condition $\psi_{\mathrm{d}}(0)=1$ follows from (1.5), and the last boundary condition follows from letting $u \downarrow 0$ in (3.9).

We remark that the integrodifferential equations (3.1), (3.4), and (3.9) satisfy relation (1.4), as do the boundary conditions (3.2), (3.5), and (3.10).

\section{Ruin with exponential claim sizes}

Paulsen and Gjessing (1997) derived explicit solutions of $\psi(u)$ when claim sizes are exponentially distributed. In this section, we derive explicit expressions for $\psi_{\mathrm{s}}(u)$ and $\psi_{\mathrm{d}}(u)$ when claim sizes are exponentially distributed. We first derive the third-order differential equations satisfied by the ruin probabilities.

Corollary 4.1. Under the conditions of Theorems 3.1, 3.2, and 3.3, if $F$ is an exponential distribution with a density function $f(x)=\beta \mathrm{e}^{-\beta x}, x>0, \beta>0$, then, for any $u>0, \psi(u)$, $\psi_{\mathrm{s}}(u)$, and $\psi_{\mathrm{d}}(u)$ satisfy the third-order differential equations

$$
\left\{\begin{array}{l}
\frac{1}{2} \sigma^{2} \psi^{\prime \prime \prime}(u)+\left(\delta u+c+\frac{1}{2} \beta \sigma^{2}\right) \psi^{\prime \prime}(u)+(\beta(\delta u+c)-\lambda+\delta) \psi^{\prime}(u)=0, \\
\frac{1}{2} \sigma^{2} \psi_{\mathrm{s}}^{\prime \prime \prime}(u)+\left(\delta u+c+\frac{1}{2} \beta \sigma^{2}\right) \psi_{\mathrm{s}}^{\prime \prime}(u)+(\beta(\delta u+c)-\lambda+\delta) \psi_{\mathrm{s}}^{\prime}(u)=0, \\
\frac{1}{2} \sigma^{2} \psi_{\mathrm{d}}^{\prime \prime \prime}(u)+\left(\delta u+c+\frac{1}{2} \beta \sigma^{2}\right) \psi_{\mathrm{d}}^{\prime \prime}(u)+(\beta(\delta u+c)-\lambda+\delta) \psi_{\mathrm{d}}^{\prime}(u)=0,
\end{array}\right.
$$

with the following boundary conditions:

$$
\begin{aligned}
\psi(\infty) & =0, & \psi(0) & =1, & & \frac{1}{2} \sigma^{2} \psi^{\prime \prime}\left(0^{+}\right)+c \psi^{\prime}\left(0^{+}\right)=0, \\
\psi_{\mathrm{s}}(\infty) & =0, & \psi_{\mathrm{s}}(0) & =0, & & \frac{1}{2} \sigma^{2} \psi_{\mathrm{s}}^{\prime \prime}\left(0^{+}\right)+c \psi_{\mathrm{s}}^{\prime}\left(0^{+}\right)=-\lambda, \\
\psi_{\mathrm{d}}(\infty) & =0, & \psi_{\mathrm{d}}(0) & =1, & & \frac{1}{2} \sigma^{2} \psi_{\mathrm{d}}^{\prime \prime}\left(0^{+}\right)+c \psi_{\mathrm{d}}^{\prime}\left(0^{+}\right)=\lambda .
\end{aligned}
$$


Proof. When $F$ is exponential, (3.9) can be re-expressed as

$$
\begin{aligned}
\lambda \psi_{\mathrm{d}}(u)-(\delta u+c) \psi_{\mathrm{d}}^{\prime}(u)-\frac{1}{2} \sigma^{2} \psi_{\mathrm{d}}^{\prime \prime}(u) & =\lambda \int_{0}^{u} \psi_{\mathrm{d}}(u-y) \mathrm{d} F(y) \\
& =\lambda \beta \mathrm{e}^{-\beta u} \int_{0}^{u} \mathrm{e}^{\beta y} \psi_{\mathrm{d}}(y) \mathrm{d} y
\end{aligned}
$$

Taking derivatives with respect to $u$ on both sides of (4.4), we obtain

$$
\begin{gathered}
\lambda \psi_{\mathrm{d}}^{\prime}(u)-\delta \psi_{\mathrm{d}}^{\prime}(u)-(\delta u+c) \psi_{\mathrm{d}}^{\prime \prime}(u)-\frac{1}{2} \sigma^{2} \psi_{\mathrm{d}}^{\prime \prime}(u) \\
=-\beta\left[\lambda \beta \mathrm{e}^{-\beta u} \int_{0}^{u} \mathrm{e}^{\beta y} \psi_{\mathrm{d}}(y) \mathrm{d} y\right]+\lambda \beta \psi_{\mathrm{d}}(u),
\end{gathered}
$$

which, together with (4.4), gives

$$
\begin{aligned}
& (\lambda-\delta) \psi_{\mathrm{d}}^{\prime}(u)-(\delta u+c) \psi_{\mathrm{d}}^{\prime \prime}(u)-\frac{1}{2} \sigma^{2} \psi_{\mathrm{d}}^{\prime \prime}(u) \\
& \quad=-\beta\left[\lambda \psi_{\mathrm{d}}(u)-(\delta u+c) \psi_{\mathrm{d}}^{\prime}(u)-\frac{1}{2} \sigma^{2} \psi_{\mathrm{d}}^{\prime \prime}(u)\right]+\lambda \beta \psi_{\mathrm{d}}(u),
\end{aligned}
$$

implying that the equation for $\psi_{\mathrm{d}}(u)$ in (4.1) holds.

The boundary conditions for $\psi_{\mathrm{d}}(u)$ follow from (3.10). The third-order differential equations for $\psi(u)$ and $\psi_{\mathrm{s}}(u)$ can be derived similarly.

We note that, when the claim sizes are exponentially distributed, the three ruin probabilities $\psi(u), \psi_{\mathrm{s}}(u)$, and $\psi_{\mathrm{d}}(u)$ satisfy the same third-order differential equation, but have different boundary conditions. The third-order differential equations in Corollary 4.1, together with the boundary conditions, enable us to obtain explicit solutions for the ruin probabilities. To do so, we recall some results about the confluent hypergeometric function and Kummer's confluent hypergeometric equation.

Assume that $y \equiv y(x)$ is twice differentiable. The following second-order differential equation is called Kummer's confluent hypergeometric equation:

$$
x \frac{\mathrm{d}^{2} y}{\mathrm{~d} x^{2}}+(b-x) \frac{\mathrm{d} y}{\mathrm{~d} x}-a y=0 .
$$

If $y_{1}$ and $y_{2}$ are any two linearly independent solutions then the general solution of (4.5) is given by

$$
y(x)=A y_{1}(x)+B y_{2}(x),
$$

where $A$ and $B$ are arbitrary coefficients that may depend on $a$ and $b$ but not on $x$, and can be determined from the boundary conditions satisfied by $y(x)$.

Denote the confluent hypergeometric function by $M(a, b ; x)$, i.e.

$$
M(a, b ; x)=\sum_{n=0}^{\infty} \frac{(a)_{n}}{(b)_{n}}\left(\frac{x^{n}}{n !}\right),
$$

where $(a)_{n}=a(a+1) \cdots(a+n-1)$ for $n=1,2, \ldots$, and $(a)_{0}=1$. 
$M(a, b ; x)$ is the simplest solution of Kummer's confluent hypergeometric equation (4.5) and is absolutely convergent for all real or complex values of $a, b$, and $x$, excluding $b=$ $0,-1,-2, \ldots$ Moreover, it is known that the confluent hypergeometric function satisfies

$$
\frac{\mathrm{d}}{\mathrm{d} x} M(a, b ; x)=\frac{a}{b} M(a+1, b+1 ; x)
$$

see Seaborn (1991) or Slater (1960) for details.

The third-order differential equations in Corollary 4.1 can be reduced to the form of Kummer's confluent hypergeometric equation using suitable function transformations. We employ the same transforms used for $\psi(u)$ in Paulsen and Gjessing (1997), since $\psi_{\mathrm{s}}(u)$ and $\psi_{\mathrm{d}}(u)$ satisfy the same third-order differential equation as does $\psi(u)$. To do so, let $u=z-\alpha$ and $\psi_{\mathrm{s}}^{\prime}(u)=\mathrm{e}^{-\beta z} g(z)$, where

$$
\alpha=\frac{c}{\delta}-\frac{\beta \sigma^{2}}{2 \delta} .
$$

Then the equation for $\psi_{\mathrm{s}}(u)$ in (4.1) becomes

$$
\frac{1}{2} \sigma^{2} g^{\prime \prime}(z)+\delta z g^{\prime}(z)+(\delta-\lambda) g(z)=0 .
$$

Furthermore, let $z^{2}=-\left(\sigma^{2} / \delta\right) x$ and $g(z)=h(x)$. Then (4.7) becomes the following Kummer confluent hypergeometric equation, with $a=\frac{1}{2}-\frac{1}{2} \lambda / \delta$ and $b=\frac{1}{2}$ :

$$
x h^{\prime \prime}(x)+\left(\frac{1}{2}-x\right) h^{\prime}(x)-\left(\frac{1}{2}-\frac{\lambda}{2 \delta}\right) h(x)=0 .
$$

Thus, by Equation (1.3.3) of Slater (1960),

$$
y_{1}(x)=\mathrm{e}^{x} U(b-a, b ;-x)=\mathrm{e}^{x} U\left(\frac{\lambda}{2 \delta}, \frac{1}{2} ;-x\right)
$$

is a solution of (4.8), where $U(a, b ; x)$ is the second form of the confluent hypergeometric function. Furthermore, by Equation (1.2.17) of Slater (1960),

$$
y_{0}(x)=x^{1-b} \mathrm{e}^{x} M(1-a, 2-b ;-x)=\sqrt{x} \mathrm{e}^{x} M\left(\frac{1}{2}+\frac{\lambda}{2 \delta}, \frac{3}{2} ;-x\right)
$$

is a solution of (4.8) and $y_{0}$ is linearly independent of $y_{1}$.

It is easy to see that if $y_{0} \equiv y_{0}(x)$ is a solution of (4.8), then so is $\sqrt{-1} y_{0}=:$ i $y_{0}$. Hence,

$$
y_{2} \equiv y_{2}(x)=\mathrm{i} y_{0}(x)=\sqrt{-x} \mathrm{e}^{x} M\left(\frac{1}{2}+\frac{\lambda}{2 \delta}, \frac{3}{2} ;-x\right)
$$

is also a solution of (4.8) linearly independent of $y_{1}$, since $y_{1}$ and $y_{0}$ are linearly independent. Therefore, the general solution to (4.8) is given by

$$
\begin{aligned}
h(x) & =A_{\mathrm{s}} y_{1}(x)+B_{\mathrm{s}} y_{2}(x) \\
& =A_{\mathrm{s}} \mathrm{e}^{x} U\left(\frac{\lambda}{2 \delta}, \frac{1}{2} ;-x\right)+B_{\mathrm{s}} \sqrt{-x} \mathrm{e}^{x} M\left(\frac{1}{2}+\frac{\lambda}{2 \delta}, \frac{3}{2} ;-x\right),
\end{aligned}
$$

where $A_{\mathrm{s}}$ and $B_{\mathrm{s}}$ are arbitrary constants. 
Thus,

$$
\begin{aligned}
g(z)=h(x)= & A_{\mathrm{s}} \mathrm{e}^{-\delta z^{2} / \sigma^{2}} U\left(\frac{\lambda}{2 \delta}, \frac{1}{2} ; \frac{\delta z^{2}}{\sigma^{2}}\right) \\
& +B_{\mathrm{s}} \frac{\sqrt{\delta} z}{\sigma} \mathrm{e}^{-\delta z^{2} / \sigma^{2}} M\left(\frac{1}{2}+\frac{\lambda}{2 \delta}, \frac{3}{2} ; \frac{\delta z^{2}}{\sigma^{2}}\right),
\end{aligned}
$$

which gives

$$
\begin{aligned}
\psi_{\mathrm{s}}^{\prime}(u)=\mathrm{e}^{-\beta z} g(z)= & A_{\mathrm{s}} \mathrm{e}^{-\beta(u+\alpha)} \exp \left\{-\frac{\delta(u+\alpha)^{2}}{\sigma^{2}}\right\} U\left(\frac{\lambda}{2 \delta}, \frac{1}{2} ; \frac{\delta(u+\alpha)^{2}}{\sigma^{2}}\right) \\
& +B_{\mathrm{s}} \mathrm{e}^{-\beta(u+\alpha)} \frac{\sqrt{\delta(u+\alpha)}}{\sigma} \exp \left\{-\frac{\delta(u+\alpha)^{2}}{\sigma^{2}}\right\} \\
& \times M\left(\frac{1}{2}+\frac{\lambda}{2 \delta}, \frac{3}{2} ; \frac{\delta(u+\alpha)^{2}}{\sigma^{2}}\right) \\
= & A_{\mathrm{s}}^{*} h_{1}(u)+B_{\mathrm{s}}^{*} h_{2}(u),
\end{aligned}
$$

where $A_{\mathrm{s}}^{*}=A_{\mathrm{s}} \mathrm{e}^{-\beta \alpha}$ and $B_{\mathrm{s}}^{*}=B_{\mathrm{s}} \sqrt{\delta} \mathrm{e}^{-\beta \alpha} / \sigma$ are arbitrary constants,

$$
h_{1}(u)=\exp \left\{-\left(\beta u+\frac{\delta(u+\alpha)^{2}}{\sigma^{2}}\right)\right\} U\left(\frac{\lambda}{2 \delta}, \frac{1}{2} ; \frac{\delta(u+\alpha)^{2}}{\sigma^{2}}\right),
$$

and

$$
h_{2}(u)=(u+\alpha) \exp \left\{-\left(\beta u+\frac{\delta(u+\alpha)^{2}}{\sigma^{2}}\right)\right\} M\left(\frac{1}{2}+\frac{\lambda}{2 \delta}, \frac{3}{2} ; \frac{\delta(u+\alpha)^{2}}{\sigma^{2}}\right) .
$$

Hence,

$$
\psi_{\mathrm{s}}(u)=-\int_{u}^{\infty} \psi_{\mathrm{s}}^{\prime}(y) \mathrm{d} y=-A_{\mathrm{s}}^{*} H_{1}(u)-B_{\mathrm{s}}^{*} H_{2}(u)
$$

where

$$
H_{1}(u)=\int_{u}^{\infty} h_{1}(y) \mathrm{d} y, \quad H_{2}(u)=\int_{u}^{\infty} h_{2}(y) \mathrm{d} y,
$$

and the constants $A_{\mathrm{s}}^{*}$ and $B_{\mathrm{s}}^{*}$ can be determined from the boundary conditions satisfied by $\psi_{\mathrm{s}}(u)$.

To determine the coefficients $A_{\mathrm{s}}^{*}$ and $B_{\mathrm{s}}^{*}$, note that $H_{1}^{\prime}(u)=-h_{1}(u)$ and $H_{2}^{\prime}(u)=-h_{2}(u)$ and, hence, $H_{1}^{\prime \prime}(u)=-h_{1}^{\prime}(u)$ and $H_{2}^{\prime \prime}(u)=-h_{2}^{\prime}(u)$. Furthermore, from (4.6), (4.9), (4.10), and

$$
\frac{\mathrm{d}}{\mathrm{d} x} U(a, b ; x)=-a U(a+1, b+1 ; x),
$$

we obtain

$$
\begin{aligned}
h_{1}^{\prime}(0)= & -\left(\beta+\frac{2 \delta \alpha}{\sigma^{2}}\right) \mathrm{e}^{-\delta \alpha^{2} / \sigma^{2}} U\left(\frac{\lambda}{2 \delta}, \frac{1}{2} ; \frac{\delta \alpha^{2}}{\sigma^{2}}\right) \\
& -\frac{\lambda \alpha}{\sigma^{2}} \mathrm{e}^{-\delta \alpha^{2} / \sigma^{2}} U\left(1+\frac{\lambda}{2 \delta}, \frac{3}{2} ; \frac{\delta \alpha^{2}}{\sigma^{2}}\right)
\end{aligned}
$$


and

$$
\begin{aligned}
h_{2}^{\prime}(0)= & \mathrm{e}^{-\delta \alpha^{2} / \sigma^{2}} M\left(\frac{1}{2}+\frac{\lambda}{2 \delta}, \frac{3}{2} ; \frac{\delta \alpha^{2}}{\sigma^{2}}\right) \\
& -\alpha\left(\beta+\frac{2 \delta \alpha}{\sigma^{2}}\right) \mathrm{e}^{-\delta \alpha^{2} / \sigma^{2}} M\left(\frac{1}{2}+\frac{\lambda}{2 \delta}, \frac{3}{2} ; \frac{\delta \alpha^{2}}{\sigma^{2}}\right) \\
& +\frac{2 \alpha^{2}(\delta+\lambda)}{3 \sigma^{2}} \mathrm{e}^{-\delta \alpha^{2} / \sigma^{2}} M\left(\frac{3}{2}+\frac{\lambda}{2 \delta}, \frac{5}{2} ; \frac{\delta \alpha^{2}}{\sigma^{2}}\right) .
\end{aligned}
$$

Thus,

$$
H_{1}^{\prime \prime}(0)=-h_{1}^{\prime}(0)=\left[\left(\beta+\frac{2 \delta \alpha}{\sigma^{2}}\right) U\left(\frac{\lambda}{2 \delta}, \frac{1}{2} ; \frac{\delta \alpha^{2}}{\sigma^{2}}\right)+\frac{\lambda \alpha}{\sigma^{2}} U\left(1+\frac{\lambda}{2 \delta}, \frac{3}{2} ; \frac{\delta \alpha^{2}}{\sigma^{2}}\right)\right] \mathrm{e}^{-\delta \alpha^{2} / \sigma^{2}}
$$

and

$$
\begin{aligned}
H_{2}^{\prime \prime}(0)=-h_{2}^{\prime}(0)= & {\left[\alpha\left(\beta+\frac{2 \delta \alpha}{\sigma^{2}}\right)-1\right] M\left(\frac{1}{2}+\frac{\lambda}{2 \delta}, \frac{3}{2} ; \frac{\delta \alpha^{2}}{\sigma^{2}}\right) \mathrm{e}^{-\delta \alpha^{2} / \sigma^{2}} } \\
& -\frac{2 \alpha^{2}(\delta+\lambda)}{3 \sigma^{2}} M\left(\frac{3}{2}+\frac{\lambda}{2 \delta}, \frac{5}{2} ; \frac{\delta \alpha^{2}}{\sigma^{2}}\right) \mathrm{e}^{-\delta \alpha^{2} / \sigma^{2}} .
\end{aligned}
$$

Moreover,

$$
H_{1}^{\prime}(0)=-h_{1}(0)=-\mathrm{e}^{-\delta \alpha^{2} / \sigma^{2}} U\left(\frac{\lambda}{2 \delta}, \frac{1}{2} ; \frac{\delta \alpha^{2}}{\sigma^{2}}\right)
$$

and

$$
H_{2}^{\prime}(0)=-h_{2}(0)=-\alpha \mathrm{e}^{-\delta \alpha^{2} / \sigma^{2}} M\left(\frac{1}{2}+\frac{\lambda}{2 \delta}, \frac{3}{2} ; \frac{\delta \alpha^{2}}{\sigma^{2}}\right) .
$$

From (4.2) and (4.11), the last two boundary conditions for $\psi_{\mathrm{s}}(u)$ reduce to

$$
\begin{gathered}
A_{\mathrm{s}}^{*} H_{1}(0)+B_{\mathrm{s}}^{*} H_{2}(0)=0, \\
\frac{1}{2} \sigma^{2}\left(A_{\mathrm{s}}^{*} H_{1}^{\prime \prime}(0)+B_{\mathrm{s}}^{*} H_{2}^{\prime \prime}(0)\right)+c\left(A_{\mathrm{s}}^{*} H_{1}^{\prime}(0)+B_{\mathrm{s}}^{*} H_{2}^{\prime}(0)\right)=\lambda .
\end{gathered}
$$

Thus, by solving the equations, we obtain

$$
A_{\mathrm{s}}^{*}=\frac{\lambda H_{2}(0) \mathrm{e}^{\delta \alpha^{2} / \sigma^{2}}}{D_{1}} \quad \text { and } \quad B_{\mathrm{s}}^{*}=\frac{-\lambda H_{1}(0) \mathrm{e}^{\delta \alpha^{2} / \sigma^{2}}}{D_{1}}
$$

where

$$
\begin{aligned}
D_{1}= & H_{1}(0) \frac{\sigma^{2}}{2} M\left(\frac{1}{2}+\frac{\lambda}{2 \delta}, \frac{3}{2} ; \frac{\delta \alpha^{2}}{\sigma^{2}}\right)+H_{1}(0) \frac{\alpha^{2}(\delta+\lambda)}{3} M\left(\frac{3}{2}+\frac{\lambda}{2 \delta}, \frac{5}{2} ; \frac{\delta \alpha^{2}}{\sigma^{2}}\right) \\
& +H_{2}(0) \frac{\lambda \alpha}{2} U\left(1+\frac{\lambda}{2 \delta}, \frac{3}{2} ; \frac{\delta \alpha^{2}}{\sigma^{2}}\right) .
\end{aligned}
$$

Similarly, the ruin probability $\psi_{\mathrm{d}}(u)$ takes the form

$$
\psi_{\mathrm{d}}(u)=-\int_{u}^{\infty} \psi_{\mathrm{d}}^{\prime}(y) \mathrm{d} y=-A_{\mathrm{d}} H_{1}(u)-B_{\mathrm{d}} H_{2}(u)
$$


where the constants $A_{\mathrm{d}}$ and $B_{\mathrm{d}}$ can be determined from the boundary conditions satisfied by $\psi_{\mathrm{d}}(u)$. In doing so, by (4.3) and (4.12), the last two boundary conditions for $\psi_{\mathrm{d}}(u)$ reduce to

$$
\begin{gathered}
A_{\mathrm{d}} H_{1}(0)+B_{\mathrm{d}} H_{2}(0)=-1, \\
\frac{1}{2} \sigma^{2}\left(A_{\mathrm{d}} H_{1}^{\prime \prime}(0)+B_{\mathrm{d}} H_{2}^{\prime \prime}(0)\right)+c\left(A_{\mathrm{d}} H_{1}^{\prime}(0)+B_{\mathrm{d}} H_{2}^{\prime}(0)\right)=-\lambda .
\end{gathered}
$$

Thus, the coefficients $A_{\mathrm{d}}$ and $B_{\mathrm{d}}$ for $\psi_{\mathrm{d}}(u)$ are given by

$$
\begin{gathered}
A_{\mathrm{d}}=\frac{1}{D_{1}}\left(-\lambda H_{2}(0) \mathrm{e}^{\delta \alpha^{2} / \sigma^{2}}-\frac{1}{2} \sigma^{2} M\left(\frac{1}{2}+\frac{\lambda}{2 \delta}, \frac{3}{2} ; \frac{\delta \alpha^{2}}{\sigma^{2}}\right)\right. \\
\left.-\frac{1}{3} \alpha^{2}(\delta+\lambda) M\left(\frac{3}{2}+\frac{\lambda}{2 \delta}, \frac{5}{2} ; \frac{\delta \alpha^{2}}{\sigma^{2}}\right)\right)
\end{gathered}
$$

and

$$
B_{\mathrm{d}}=\frac{1}{D_{1}}\left(-\frac{1}{2} \lambda \alpha U\left(1+\frac{\lambda}{2 \delta}, \frac{3}{2} ; \frac{\delta \alpha^{2}}{\sigma^{2}}\right)+\lambda H_{1}(0) \mathrm{e}^{\delta \alpha^{2} / \sigma^{2}}\right) .
$$

To end the paper, we use numerical examples to illustrate applications of the explicit solutions and the effects of the interest force $\delta$ and the diffusion volatility $\sigma$ on the ruin probabilities $\psi(u)$, $\psi_{\mathrm{s}}(u)$, and $\psi_{\mathrm{d}}(u)$.

Example 4.1. Assume that the claim sizes are exponentially distributed and that $c=103$, $\lambda=100, \beta=1$, and $\sigma=0.8$. We calculate the values of $\psi(u), \psi_{\mathrm{s}}(u)$, and $\psi_{\mathrm{d}}(u)$, using the formulae derived above, for $\delta=0.105,0.085,0.065,0.045,0$. When $\delta=0$, by Equations (6.4) and (6.17) of Dufresne and Gerber (1991), we have

$$
\begin{aligned}
\psi(u) & =c_{1} \mathrm{e}^{-r_{1} u}+c_{2} \mathrm{e}^{-r_{2} u}, \\
\psi_{\mathrm{s}}(u) & =c_{1}^{\mathrm{s}} \mathrm{e}^{-r_{1} u}+c_{2}^{\mathrm{s}} \mathrm{e}^{-r_{2} u}, \\
\psi_{\mathrm{d}}(u) & =c_{1}^{\mathrm{d}} \mathrm{e}^{-r_{1} u}+c_{2}^{\mathrm{d}} \mathrm{e}^{-r_{2} u},
\end{aligned}
$$

where

$$
\begin{aligned}
c_{1} & =\frac{r_{1}-\beta}{\beta} \frac{r_{2}}{r_{1}-r_{2}}, & c_{2} & =\frac{r_{2}-\beta}{\beta} \frac{r_{1}}{r_{2}-r_{1}}, \\
c_{1}^{\mathrm{s}} & =c_{1}-c_{1}^{\mathrm{d}}, & c_{2}^{\mathrm{s}} & =c_{2}-c_{2}^{\mathrm{d}}, \\
c_{1}^{\mathrm{d}} & =\frac{r_{1}-\beta}{r_{1}-r_{2}}, & c_{2}^{\mathrm{d}} & =\frac{r_{2}-\beta}{r_{2}-r_{1}},
\end{aligned}
$$

and $r_{1}$ and $r_{2}$ are the solutions to the equation $\lambda /(\beta-r)+\frac{1}{2} \sigma^{2} r=c$.

In Tables 1-3 we present the values of these ruin probabilities for selected values of $u$. The calculations were performed using MATHEMATICA ${ }^{\circledR}$. From the tables, we see that the ruin probabilities $\psi(u)$ and $\psi_{\mathrm{d}}(u)$ decrease as $u$ and $\delta$ increase and that the dependence of $\psi(u)$ on $\delta$ is strongest for small values of $u$. On the other hand, the behavior of $\psi_{\mathrm{s}}(u)$ differs from that of both $\psi(u)$ and $\psi_{\mathrm{d}}(u)$. For example, $\psi_{\mathrm{s}}(u)$ is not a decreasing function of $u$ and $\delta$.

Example 4.2. Assume that the claim sizes are exponentially distributed and that $c=103$, $\lambda=100, \beta=1$, and $\delta=0.03$. We calculate the values of $\psi(u), \psi_{\mathrm{s}}(u)$, and $\psi_{\mathrm{d}}(u)$, using the formulae derived above, for $\sigma=1.2,1.0,0.8,0.6,0.4$, and present the ruin probabilities 
TABLE 1: Effects of interest force on the ruin probability $\psi(u)$.

\begin{tabular}{lccccc}
\hline & \multicolumn{5}{c}{$\psi(u)$} \\
\cline { 2 - 6 } \multicolumn{1}{c}{$u$} & $\delta=0.105$ & $\delta=0.085$ & $\delta=0.065$ & $\delta=0.045$ & $\delta=0$ \\
\hline 0 & 1.000000 & 1.000000 & 1.000000 & 1.000000 & 1.000000 \\
0.001 & 0.987379 & 0.987993 & 0.988672 & 0.989446 & 0.991984 \\
0.005 & 0.963270 & 0.965056 & 0.967035 & 0.969284 & 0.976671 \\
0.01 & 0.955781 & 0.957931 & 0.960313 & 0.963021 & 0.971914 \\
0.055 & 0.952201 & 0.954526 & 0.957100 & 0.960027 & 0.969640 \\
1 & 0.910692 & 0.915029 & 0.919835 & 0.925300 & 0.943257 \\
5 & 0.749126 & 0.761087 & 0.774390 & 0.789574 & 0.839816 \\
10 & 0.576690 & 0.595926 & 0.617522 & 0.642414 & 0.726320 \\
50 & 0.034760 & 0.046777 & 0.063926 & 0.089242 & 0.227340 \\
\hline
\end{tabular}

TABLE 2: Effects of interest force on the ruin probability $\psi_{\mathrm{s}}(u)$.

\begin{tabular}{lccccc}
\hline & \multicolumn{5}{c}{$\psi_{\mathrm{s}}(u)$} \\
\cline { 2 - 6 } \multicolumn{1}{c}{$u$} & $\delta=0.105$ & $\delta=0.085$ & $\delta=0.065$ & $\delta=0.045$ & $\delta=0$ \\
\hline 0 & 0.000000 & 0.000000 & 0.000000 & 0.000000 & 0.000000 \\
0.001 & 0.262479 & 0.263091 & 0.263768 & 0.264539 & 0.267069 \\
0.005 & 0.761860 & 0.763641 & 0.765613 & 0.767855 & 0.775218 \\
0.01 & 0.913326 & 0.915469 & 0.917843 & 0.920543 & 0.929408 \\
0.055 & 0.949251 & 0.951569 & 0.954135 & 0.957053 & 0.966636 \\
1 & 0.907871 & 0.912195 & 0.916986 & 0.922434 & 0.940335 \\
5 & 0.746806 & 0.758730 & 0.771991 & 0.787128 & 0.837215 \\
10 & 0.574903 & 0.594080 & 0.615609 & 0.640424 & 0.724070 \\
50 & 0.034652 & 0.046632 & 0.063728 & 0.088966 & 0.226636 \\
\hline
\end{tabular}

TABLE 3: Effects of interest force on the ruin probability $\psi_{\mathrm{d}}(u)$.

\begin{tabular}{lccccc}
\hline & \multicolumn{5}{c}{$\psi_{\mathrm{d}}(u)$} \\
\cline { 2 - 6 } \multicolumn{1}{c}{$u$} & $\delta=0.105$ & $\delta=0.085$ & $\delta=0.065$ & $\delta=0.045$ & $\delta=0$ \\
\hline 0 & 1.000000 & 1.000000 & 1.000000 & 1.000000 & 1.000000 \\
0.001 & 0.724900 & 0.724902 & 0.724904 & 0.724907 & 0.724915 \\
0.005 & 0.201410 & 0.201415 & 0.201422 & 0.201429 & 0.201453 \\
0.01 & 0.042455 & 0.042462 & 0.042470 & 0.042478 & 0.042506 \\
0.055 & 0.002950 & 0.002957 & 0.002965 & 0.002974 & 0.003004 \\
1 & 0.002821 & 0.002834 & 0.002849 & 0.002866 & 0.002922 \\
5 & 0.002320 & 0.002357 & 0.002399 & 0.002446 & 0.002601 \\
10 & 0.001787 & 0.001846 & 0.001913 & 0.001990 & 0.002250 \\
50 & 0.000108 & 0.000145 & 0.000198 & 0.000276 & 0.000704 \\
\hline
\end{tabular}

for selected values of $u$ in Tables 4-6. These calculations were also performed using MATHEMATICA.

From the tables, we see that the ruin probabilities $\psi(u)$ and $\psi_{\mathrm{d}}(u)$ increase as $\sigma$ increases and that the dependence of $\psi(u)$ on $\sigma$ is strongest for small values of $u$, as in Example 4.1. Furthermore, the behavior of $\psi_{\mathrm{s}}(u)$ again differs from that of both $\psi(u)$ and $\psi_{\mathrm{d}}(u)$. 
TABLE 4: Effects of volatility on the ruin probability $\psi(u)$.

\begin{tabular}{lccccc}
\hline & \multicolumn{5}{c}{$\psi(u)$} \\
\cline { 2 - 6 } \multicolumn{1}{c}{$u$} & $\sigma=1.2$ & $\sigma=1.0$ & $\sigma=0.8$ & $\sigma=0.6$ & $\sigma=0.4$ \\
\hline 0 & 1.000000 & 1.000000 & 1.000000 & 1.000000 & 1.000000 \\
0.001 & 0.995211 & 0.993313 & 0.990116 & 0.984354 & 0.974003 \\
0.005 & 0.981630 & 0.976884 & 0.971236 & 0.966081 & 0.964039 \\
0.01 & 0.972601 & 0.968560 & 0.965372 & 0.963986 & 0.963808 \\
0.055 & 0.962845 & 0.962682 & 0.962568 & 0.962479 & 0.962416 \\
1 & 0.930389 & 0.930200 & 0.930045 & 0.929924 & 0.929837 \\
5 & 0.803475 & 0.803104 & 0.802800 & 0.802563 & 0.802393 \\
10 & 0.665251 & 0.664723 & 0.664289 & 0.663951 & 0.663708 \\
50 & 0.117848 & 0.117389 & 0.117012 & 0.116720 & 0.116511 \\
\hline
\end{tabular}

TABLE 5: Effects of volatility on the ruin probability $\psi_{\mathrm{s}}(u)$.

\begin{tabular}{lccccc}
\hline & \multicolumn{5}{c}{$\psi_{\mathrm{s}}(u)$} \\
\cline { 2 - 6 } \multicolumn{1}{c}{$u$} & $\sigma=1.2$ & $\sigma=1.0$ & $\sigma=0.8$ & $\sigma=0.6$ & $\sigma=0.4$ \\
\hline 0 & 0.000000 & 0.000000 & 0.000000 & 0.000000 & 0.000000 \\
0.001 & 0.128448 & 0.179399 & 0.265207 & 0.419898 & 0.697769 \\
0.005 & 0.491507 & 0.618604 & 0.769801 & 0.907567 & 0.961699 \\
0.01 & 0.730629 & 0.838269 & 0.922886 & 0.959069 & 0.963057 \\
0.055 & 0.955419 & 0.957999 & 0.959586 & 0.960800 & 0.961669 \\
1 & 0.923929 & 0.925706 & 0.927164 & 0.928302 & 0.929115 \\
5 & 0.797896 & 0.799224 & 0.800313 & 0.801162 & 0.801770 \\
10 & 0.660632 & 0.661511 & 0.662231 & 0.662793 & 0.663193 \\
50 & 0.117030 & 0.116821 & 0.116650 & 0.116516 & 0.116421 \\
\hline
\end{tabular}

TABLE 6: Effects of volatility on the ruin probability $\psi_{\mathrm{d}}(u)$.

\begin{tabular}{lccccc}
\hline & \multicolumn{5}{c}{$\psi_{\mathrm{d}}(u)$} \\
\cline { 2 - 6 } \multicolumn{1}{c}{$u$} & $\sigma=1.2$ & $\sigma=1.0$ & $\sigma=0.8$ & $\sigma=0.6$ & $\sigma=0.4$ \\
\hline 0 & 1.000000 & 1.000000 & 1.000000 & 1.000000 & 1.000000 \\
0.001 & 0.866763 & 0.813914 & 0.724909 & 0.564456 & 0.276234 \\
0.005 & 0.490123 & 0.358280 & 0.201435 & 0.058514 & 0.002340 \\
0.01 & 0.241972 & 0.130291 & 0.042486 & 0.004917 & 0.000751 \\
0.055 & 0.007426 & 0.004683 & 0.002982 & 0.001679 & 0.000747 \\
1 & 0.006460 & 0.004494 & 0.002881 & 0.001622 & 0.000722 \\
5 & 0.005579 & 0.003880 & 0.002487 & 0.001401 & 0.000623 \\
10 & 0.004619 & 0.003212 & 0.002058 & 0.001158 & 0.000515 \\
50 & 0.000818 & 0.000568 & 0.000362 & 0.000204 & 0.000090 \\
\hline
\end{tabular}

\section{Acknowledgements}

This research was supported by the Natural Sciences and Engineering Research Council of Canada and a grant from the Research Grants Council of the Hong Kong Special Administrative Region, China (project no. HKU 7050/05P). We also thank a referee for a careful reading of the paper and helpful suggestions that improved its presentation. 
In addition, Professor Guojing Wang recently informed us of his paper Wang (2001), in which he considered a different approach to a similar problem.

\section{References}

Asmussen, S. (2000). Ruin Probabilities. World Scientific, Singapore.

CAI, J. (2004). Ruin probabilities and penalty functions with stochastic rates of interest. Stoch. Process. Appl. 112, 53-78.

CAI, J. AND Dickson, D. C. M. (2002). On the expected discounted penalty function at ruin of a surplus process with interest. Insurance Math. Econom. 30, 389-404.

Crandall, M. G. And Lions, P. L. (1983). Viscosity solutions of Hamilton-Jacobi equations. Trans. Amer. Math. Soc. 277, 1-42.

Crandall, M. G., Ishit, H. and Lions, P.-L. (1992). User's guide to viscosity solutions of second order partial differential equations. Bull. Amer. Math. Soc. 27, 1-67.

Dufresne, F. AND Gerber, H. U. (1991). Risk theory for the compound Poisson process that is perturbed by diffusion. Insurance Math. Econom. 10, 51-59.

Fleming, W. H. ANd Soner, H. M. (1993). Controlled Markov Processes and Viscosity Solutions. Springer, New York.

Gerber, H. U. AND LANDRY, B. (1998). On the discounted penalty at ruin in a jump-diffusion and the perpetual put option. Insurance Math. Econom. 22, 263-276.

Grandell, J. (1991). Aspects of Risk Theory. Springer, New York.

HipP, C. AND Plum, M. (2003). Optimal investment for investors with state dependent income, and for insurers. Finance Stoch. 7, 299-321.

Paulsen, J. And Gjessing, H. K. (1997). Ruin theory with stochastic economic environment. Adv. Appl. Prob. 29, 965-985.

SeAborn, J. B. (1991). Hypergeometric Functions and Their Applications. Springer, New York.

Slater, L. J. (1960). Confluent Hypergeometric Functions. Cambridge University Press.

Sundt, B. And Teugels, J. L. (1995). Ruin estimates under interest force. Insurance Math. Econom. 16, 7-22.

WANG, G. (2001). A decomposition of the ruin probability for the risk process perturbed by diffusion. Insurance Math. Econom. 28, 49-59.

WANG, G. AND WU, R. (2001). Distributions for the risk process with a stochastic return on investments. Stoch. Process. Appl. 95, 329-341.

YANG, H. L. AND Zhang, L. (2001). Spectrally negative Lévy processes with applications in risk theory. Adv. Appl. Prob. 33, 281-291. 